\title{
PART II: DETERMINATION OF TOTAL DISSOLVED CARBONATE AND CARBON ISOTOPE RATIOS ${ }^{1}$
}

\author{
B. J. Presley and G. E. Claypool, Department of Geology and Institute of \\ Geophysics and Planetary Physics, University of California, Los Angeles, California
}

\begin{abstract}
Introduction
Most of the analytical techniques used in interstitial water studies at UCLA have been described in Part I of this report. Some details regarding sample handling and storage can be found there, too.
\end{abstract}

In this part of the report we describe the technique used to determine total dissolved carbonate and carbon isotope ratios. Although the procedure for removing, purifying, and collecting carbon dioxide for carbonate samples is well known, (McCrea, 1950; Sackett and Moore, 1966; Deuser and Hunt, 1969) it was necessary to significantly modify the volume measurement procedure in order to accommodate small (micromolar) quantities of carbon dioxide.

\section{Procedure}

The procedure basically consists of acidifying the sample under vacuum, then purifying and measuring the evolved carbon dioxide.

As noted in Part I, the water sample is received sealed in a 5-milliliter plastic syringe. The syringe is weighed and about 3.5 milliliters of the water are transferred to a reaction flask. The syringe is then re-weighed to determine the exact amount of water used.

This quantity ( 3.5 milliliters) of typical surface seawater will only yield about 0.2 milliliter of carbon dioxide upon acidification. Most interstitial waters contain comparable or greater amounts of total carbon dioxide, but some have much less. Thus, in many cases we have been forced to work with very small gas volumes.

One milliliter of $1 \mathrm{~N} \mathrm{HCl}$ is put into the rotatable side arm of a custom-made 50-milliliter round bottom flask, which can be closed with a stopcock. $\left(\mathrm{H}_{3} \mathrm{PO}_{4}\right.$ would be used, but interferes in the determination of sulfate in the next step in the analytical scheme.) The open flask with the side arm containing the hydrochloric acid attached is swept for 1 or 2 minutes with a vigorous stream of tank nitrogen issuing from tygon tubing extended to the bottom of the flask. We have

\footnotetext{
${ }^{1}$ Publication No. 870 Institute of Geophysics \& Planetary
} Physics. found that this sweeping effectively removes nearly all the atmospheric carbon dioxide from the flask and side arm.

The tubing is removed from the flask, the sample is introduced from the syringe, and the flask is closed with a stopcock. This operation takes only a few seconds and appears to result in an insignificant atmospheric contamination.

The flasks are assembled on the vacuum line (Figure 1) and both sample and hydrochloric acid are frozen in liquid nitrogen. After a few minutes freezing the flasks are opened and are pumped down to about 5 microns of mercury pressure. They are then closed, removed from the line, and allowed to warm to room temperature. The side arms are rotated to add the hydrochloric acid to the sample, and the flasks are shaken vigorously. They are left overnight to react, shaken again the next day, and re-assembled on the vacuum line. (We have carried out no experiments to determine how much time is actually needed for the carbon dioxide to outgas from the sample, but the time we allow is probably much longer than is necessary.)

The contents of the flasks are frozen in a dry ice-acetone slurry, which does not condense carbon dioxide. The carbon dioxide is then transferred to an adjacent trap cooled with liquid nitrogen, the noncondensable gases pumped away, and the carbon dioxide distilled twice more through dry ice-acetone to complete the separation from water. This procedure gives about a 98 per cent yield of the carbon dioxide produced in the reaction flask. This can be made to approach 100 per cent by completely thawing and then refreezing the reaction flask, but this additional step was not included in our routine procedure.

The last transfer of the carbon dioxide is made to the closed leg of the gas pipette-manometer by filling a cup at the base of the pipette with liquid nitrogen (see Figure 1). The solid carbon dioxide is confined to the pipette by raising the mercury level above the U-bend with nitrogen gas pressure. When the sample is trapped, the liquid nitrogen is blown out of the cup and the glass warmed by a stream of air. After the carbon dioxide sublimes the mercury level is raised, compressing the sample into the calibrated portion of 


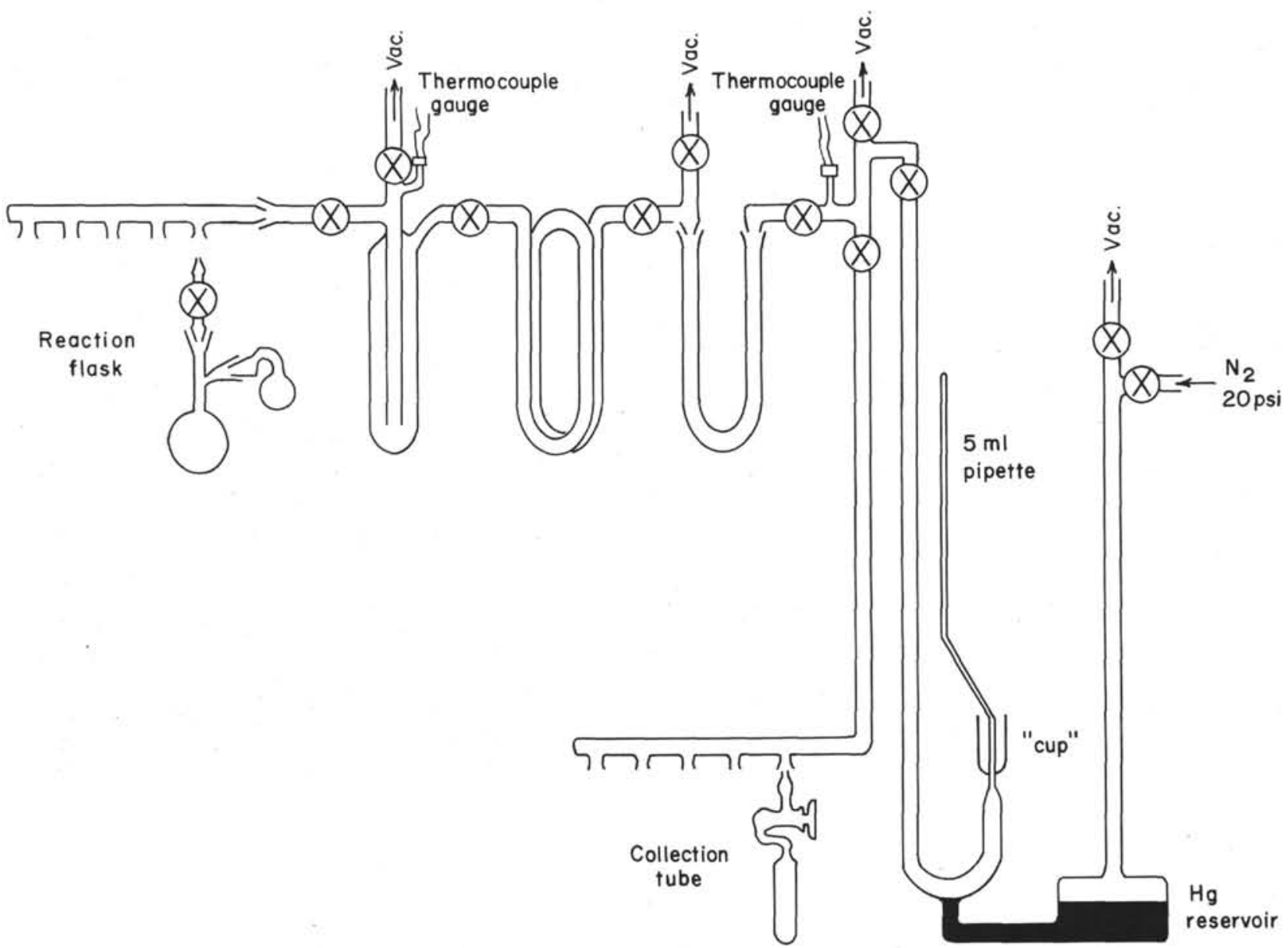

Figure 1. Vacuum line used in extraction of carbon dioxide from samples

the pipette (a 5 milliliter burette graduated in 0.02 milliliters, plugged at the zero mark).

The volume of the gas is read directly. The pressure at which the volume was read is the difference in height of the two mercury columns. A simple gas law calculation converts the volume to STP.

Obviously there is a "best" combination of pressure and volume for measuring each sample. That is, a combination that gives the least combined error in reading the pressure difference and the volume. If either is made too large, the other becomes small and difficult to determine accurately. Our policy is to raise the mercury column until we get at least 100 millimeters pressure difference, thus the pressure can be easily determined to within 1 per cent. A pressure difference of 100 millimeters means that the sample volume is roughly 7.6 times the STP volume (ignoring for the moment the much smaller temperature effect). Thus, a 0.2 milliliter sample would occupy about 1.5 milliliters, and with a good 5 milliliter burette this volume can be determined to within roughly 1 per cent.

Actually, we always measure each sample at two different pressure-volume combinations. This only takes an extra minute or so, and errors in reading or recording of values can be detected. The STP volumes calculated from different combinations seldom disagree by more than 1 per cent.

After the sample volume has been measured, the mercury level in the gas pipette-manometer is lowered by pumping away the gas used to raise it, and the carbon dioxide is collected in a sample tube for transfer to the mass spectrometer.

The carbon isotope ratio is determined with a Nuclide $60^{\circ}$, 6-inch radius dual collecting mass spectrometer. All samples are measured against a calcite substandard calibrated against the PDB standard.

\section{REFERENCES}

Deuser, W. G. and Hunt, J. M., 1969. Stable isotope ratios of dissolved inorganic carbon in the Atlantic. Deep-Sea Res. 16, 221.

McCrea, J. M., 1950. On the isotopic chemistry of carbonates and a paleotemperature scale. J. Chem. Phys. 118, 849 .

Sackett, W. M. and Moore, W. S., 1966. Isotopic variations of dissolved inorganic carbon. Chem. Geol. 1, 323. 